\title{
Zwei Tage pro Woche fasten reicht
}

\author{
Abspecken funktioniert nur über eine Änderung des Essverhaltens. Offenbar \\ muss man aber nicht unbedingt jeden Tag die Energiezufuhr reduzieren.
}

_ Reicht es beim Abnehmen, nur alle paar Tage die Kalorien reduzieren, um ein Energiedefizit zu erzielen? Dem ging eine Studie mit 115 übergewichtigen Frauen zwischen 20 und 69 Jahren nach. Eine Gruppe hielt täglich eine Reduktionsdiät mit 25\% Energiedefizit als mediterrane Kost ein (45\% Kohlenhydrate, 25\% Eiweiß, 30\% Fett).

Eine weitere Gruppe aß pro Woche fünf Tage lang eukalorisch mediterran und reduzierte dann zwei Tage lang die Energiezufuhr um 70\%. An diesen Tagen wurden vor allem Kohlenhydrate reduziert, sodass ihr Anteil auf ca. 20\% sank. Eine dritte Gruppe sollte sich verhalten wie die zweite, durfte aber an den Reduktionstagen nach Belieben bestimmte eiweiß- und fettreiche Lebensmittel zusätzlich zu sich nehmen.

Das Ziel, mindestens $5 \mathrm{~kg}$ in drei Monaten abzunehmen, erreichten in der Gruppe mit täglicher Restriktion 48\% der Frauen. In der strengen Zwei-TageGruppe waren es 65\%, in der etwas laxeren immerhin 58\%. Auch bei der korres- pondierenden Abnahme des Körperfetts und beim Taillenumfang lag die strenge Zwei-Tage-Diät vorn und die tägliche Restriktion hinten.

Auch Parameter der Insulinresistenz (Plasma-Insulin, HOMA-Index) waren unter der intermittierenden Reduktionsdiät deutlich gebessert, während die tägliche Diät keine Auswirkungen hatte.

- Harvie M et al. The effect of intermittent energy and carbohydrate restriction $v$. daily energy restriction on weight loss and metabolic disease risk markers in overweight women. Br J Nutr. 2013;110:1534-47

\section{KOMMENTAR}

Diese Ergebnisse überraschen, da frühere Studien Ähnliches bisher nicht vorweisen konnten. Der Schlüssel zum Erfolg liegt aber wahrscheinlich in der Einhaltung der Diät. Mediterrane Kost wird in der Regel von Probanden geschmacklich gut toleriert und sättigt durch den relativ hohen Anteil an Ballaststoffen ausreichend. Der vermehrte Konsum von Eiweiß und Fett hatte je keinen Vorteil, wenngleich Eiweiß verstärkt sättigt.
Pathophysiologisch ungeklärt ist, ob eine starke Energierestriktion an wenigen Tagen metabolische Mechanismen induziert, die bei mäßiger Zurückhaltung an allen Tagen nicht oder nicht so ausgeprägt auftreten. Tierversuche lassen dabei u. a. an den Intermediärstoffwechsel, Karzinome sowie die Mortalität denken.

Als Fazit bleibt: Wem es leichter fällt, zwei Tage die Woche die Nahrungsaufnahme erheblich zu vermindern statt die ganze Woche weniger zu essen, sollte es versuchen.

Prof. Dr. med. A. Wirth

\section{Hier steht eine Anzeige.}

\author{
Springer
}

